\title{
Manual de Obesidades. Una oportunidad para mejorar la salud de mi paciente
}

\author{
Leonardo Mancillas Adame
}

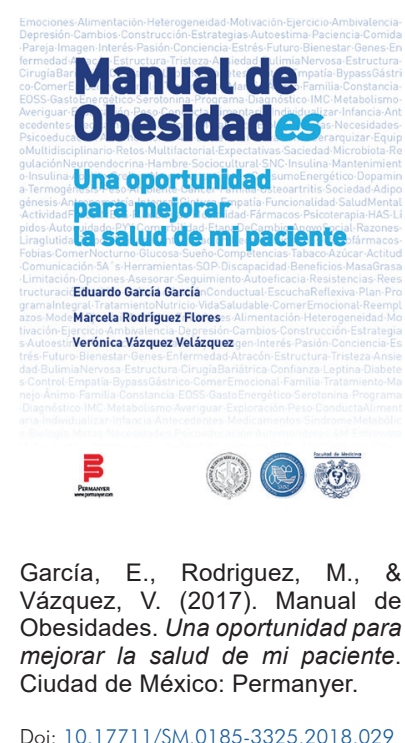

(c) (P)
El objetivo de este manual es proporcionarle al lector herramientas para diagnosticar y tratar adecuadamente a las personas que padecen obesidad. Para esto, los autores parten de la evidencia científica disponible y de la experiencia clínica desarrollada en la Clínica de Obesidad y de Trastornos de la Conducta Alimentaria del Instituto Nacional de Ciencias Médicas y Nutrición Salvador Zubirán en casi dos décadas de trabajo.

Los autores explican en la introducción: "Con el título, Obesidades una oportunidad para mejorar la salud de mi paciente, queremos señalar dos aspectos sobresalientes del diagnóstico y tratamiento de la obesidad: Primero, la heterogeneidad de la obesidad en sus expresiones clínicas, por eso nos referimos a ella como las obesidades, queriendo subrayar sus diferentes mecanismos de construcción y en consecuencia la necesidad de partir de reconocer la necesidad de mejorar nuestras habilidades diagnósticas y de clasificación, preguntándonos y respondiéndonos en cada uno de los casos que nos ocupe ¿cómo es la obesidad del paciente que tenemos frente a nosotros? En segundo lugar, queremos dejar clara la conexión entre un adecuado diagnóstico y la oportunidad que de ello se deriva para organizar una intervención exitosa, haciendo una propuesta de tratamiento ad hoc a cada caso".

Se trata de una obra que, sin ser un libro de texto, marca con precisión las acciones por realizar; pero que proporciona, además, una explicación del porqué y del para qué de estas acciones. Doscientas ochenta páginas que abordan las distintas perspectivas del diagnóstico y tratamiento multidisciplinario que hoy exige la obesidad en el adulto. Si bien surgió por la necesidad de dotar al médico de primer contacto -quien en sus años de formación curricular tiene escaso contacto con las estrategias vigentes de diagnóstico y tratamiento de la obesidad- de herramientas para que pueda sentirse seguro en su deseo y necesidad de ayudar a los pacientes que padecen esta enfermedad, también resulta de gran utilidad para el nutriólogo, el psicólogo y el psiquiatra, los cuales en su quehacer diario tienen contacto con estos pacientes. Más aún, enfatiza la conveniencia de las estrategias multidisciplinarias.

En el capítulo 1, La heterogeneidad de la obesidad y el reto clínico de su tratamiento, se pretende que el lector revise los distintos escenarios y tipos de obesidad que se identifican con frecuencia en la práctica clínica, para que con esto mejore el proceder diagnóstico y terapéutico según las particularidades del paciente, teniendo en cuenta no sólo factores físicos y médicos, sino también factores psicológicos y emocionales.

El reconocimiento de la obesidad como una enfermedad por parte de los profesionales de la salud, los pacientes y la sociedad es una prioridad. En el capítulo 2, La obesidad: Definición, fisiopatogenia y distintas percepciones, se abordan precisamente los determinantes de la obesidad, considerando los aspectos neurobiológicos la alimentación, la regulación homeostática, la dinámica energética a lo largo de los cambios en el peso, entre otros temas. Información indispensable para la comprensión de esta enfermedad tan compleja.

El capítulo 3, que habla sobre el diagnóstico de la obesidad, explica los criterios para el diagnóstico antropométrico y de composición corporal, así como la utilidad de diferentes herramientas para integrar sistemáticamente un diagnóstico médico, mental y funcional para plantear el beneficio de la reducción de peso en los riesgos y daños a la salud de forma individual. 
De este modo, el apartado de Diagnóstico Integral de la Obesidad está conformado por seis componentes. El automonitoreo, enfatiza que éste es el más importante instrumento para el diagnóstico y tratamiento que, bien utilizado, fomenta el cambio conductual. Para ello, el médico debe saber transmitir al paciente la idea de que no es un método para calificarlo, sino que es una herramienta de comunicación entre el paciente y el médico, un método de autoexploración que le permitirá conocerse mejor y recibir ayuda más eficaz. Se muestran distintos formatos de automonitoreo, las variables que registran y los perfiles de pacientes en los que cada uno estaría más indicado.

Se describe con detalle la Escala de Estadificación de Obesidad de Edmonton (EOSS, Edmonton Obesity Staging scale), instrumento incluido en la mayoría de las distintas guías clínicas para el tratamiento de la obesidad y que se ha venido convirtiendo en una clasificación indispensable para precisar las afecciones de cada paciente. El autor de este sistema, el Dr. Arya Sharma, participó en la elaboración de este apartado del manual. La intención del EOSS es clasificar a los pacientes con sobrepeso u obesidad (Índice de Masa Corporal mayor o igual de 25), considerando no sólo dicho valor sino incorporando sistemáticamente las diferentes herramientas diagnósticas en un diagnóstico médico, mental y funcional para determinar el beneficio de la reducción de peso en los riesgos y daños a la salud de forma individual. La idea es aplicar un esquema tridimensional de salud que proporcione un esquema terapéutico apropiado para el tipo de obesidad, incluyendo la necesidad de tratamientos más intensivos, referencias y tratamientos adyuvantes en conjunto con dieta y ejercicio.

Con el fin de lograr este esquema tridimensional, hay tres capítulos que muestran las herramientas para el diagnóstico integral: De lo médico (comorbilidades médicas de la obesidad, procedimientos diagnósticos clínicos, de laboratorio y gabinetes para el estudio de los pacientes con obesidad), De lo funcional (trastornos articulares más comúnmente asociados con la obesidad, aspectos de movilidad y su asociación con el pronóstico y la calidad de vida en individuos con obesidad) y De lo psicológico y psiquiátrico (aspectos de estos ámbitos que pueden estar presentes en algunos pacientes con obesidad). En todos estos capítulos se proporcionan herramientas al profesional de la salud para aproximarse al diagnóstico (criterios, formatos, cuestionarios, entrevistas, etc.). Finalmente, el capítulo Integración del diagnóstico presenta casos clínicos y formatos para aplicar los conocimientos adquiridos en los capítulos anteriores y de llevar a cabo un diagnóstico integral del paciente con obesidad. Todo lo anterior se aterriza en el análisis de casos clínicos construidos como ejercicios para aplicar las estrategias de diagnóstico que permite al lector alcanzar una nueva habilidad.

Después del planteamiento del diagnóstico, se incluyen dos capítulos que permiten revisar el lenguaje, la comuni- cación y las habilidades del profesional de la salud para transmitir los mensajes, aspectos en los que se basan las propuestas actuales de tratamiento para pacientes que padecen enfermedades crónicas.

En el capítulo Habilidades de Comunicación, se evalúan las capacidades de comunicación del profesional de la salud con el fin de mantener o mejorar sus habilidades para comunicarse de forma exitosa con el paciente, así como saber qué lenguaje usar y qué lenguaje evitar. Además, se pretende que el lector sea capaz de utilizar recursos para comprender al paciente, conocer los factores causantes de la obesidad, proporcionar el asesoramiento necesario y ayudar al paciente a alcanzar sus metas. Cabe mencionar que estas herramientas están basadas en el modelo de las 5 A's, que fue diseñado originalmente como una estrategia de intervención conductual para dejar de fumar. El modelo fue modificado para el tratamiento de la obesidad para que los profesionales de la salud lo utilizaran como un marco de referencia para dirigir la conversación. Este modelo se ha asociado con un incremento en la motivación del paciente y en los cambios conductuales cuando se utiliza por los médicos en su consulta.

En el capítulo Estrategias de la Entrevista Motivacional, se explica que este tipo de entrevista clínica directiva, centrada en el paciente, tiene como objetivo incrementar la motivación intrínseca para el cambio hacia un estilo de vida más saludable, mediante la exploración y la resolución de la ambivalencia que esto provoca. Se proporciona al lector una manera práctica para recordar dichas estrategias y para incluirlas en la consulta con el fin de favorecer la motivación de los pacientes.

La segunda parte del manual está enfocado al tratamiento. En el capítulo Elaboración del plan de tratamiento se invita al lector a determinar sus objetivos para lograr un beneficio significativo en el paciente, aplicando un algoritmo de tratamiento, a partir de las recomendaciones de las guías para el tratamiento de la obesidad. Nuevamente se exponen casos clínicos de vida real para otorgar un sentido práctico a las propuestas del manual, al mismo tiempo que se realiza el ejercicio de diagnóstico con el EOSS y las sugerencias que cada profesional de la salud le daría a cada paciente.

Se realiza un recorrido por las distintas intervenciones aprobadas para el tratamiento no farmacológico, farmacológico y quirúrgico. Dentro de las opciones de tratamiento no farmacológico, se incluyen el capítulo Opciones del tratamiento nutricio, que ofrece al médico de primer contacto las herramientas básicas para aproximarse a una adecuada evaluación, diagnóstico y prescripción nutricia, tomando en cuenta la limitación de tiempo y recursos que pueden tener tanto él como sus pacientes. Se incluye también el capítulo Opciones de actividad física, donde se habla de los tipos de ejercicio y sus indicaciones según el perfil del paciente, además de los beneficios del ejercicio en el paciente 
con obesidad y las precauciones que deben adoptarse, y el capítulo Terapia cognitivo conductual, para conocer su aplicación en el tratamiento de la obesidad y algunas estrategias cognitivo-conductuales, sus elementos clave y sus objetivos.

Hay cuatro capítulos de tratamiento farmacológico. Uno que habla de los fármacos para la obesidad, aprobados para el tratamiento de la obesidad a largo plazo, sus distintos mecanismos de acción, indicaciones y precauciones de uso. Otro capítulo que habla de los psicofármacos en el tratamiento de los pacientes con obesidad, enfocado a que el lector adquiera la competencia de conocer los principales trastornos psiquiátricos que pueden coexistir con la obesidad, de saber determinar la gravedad de estos trastornos y si es necesario iniciar tratamiento psicofarmacológico y conocer los psicofármacos indicados en cada caso, sus beneficios y sus posibles efectos adversos. Además, se incluyen dos capítulos de fármacos que producen aumento de peso y fármacos que no deben usarse en el tratamiento del paciente con obesidad.

Finalmente, hay tres capítulos de cirugía bariátrica: A. Indicaciones y tipos de cirugía, B. Preparación del paciente, C. Seguimiento. En estos capítulos se proporcionan los criterios para que a un paciente con obesidad se le ofrezca, además del tratamiento médico-nutricio-psicológico, la opción de cirugía bariátrica. Se mencionan los tipos, las ventajas y desventajas de cada procedimiento, el proceso de preparación del paciente y su red de apoyo, así como la importancia del seguimiento para toda la vida, de las comorbilidades, deficiencias, salud mental y calidad de vida.

En el capítulo 8, Canalización del paciente con otro profesional, se habla sobre la necesidad de saber identificar las situaciones en las que está indicado referir al paciente a otros profesionales y cómo hacerlo.

Los múltiples ejercicios que el manual propone al lector concluyen con la propuesta de elaborar una estrategia o programa "para mi práctica clínica", con la finalidad de entregar al lector un producto final que mejore su práctica diaria (capítulo 9, Elaborando una estrategia o programa para mi práctica clínica). La intención es que el lector sea capaz de establecer una estrategia individualizada tomando en cuenta la naturaleza compleja de la obesidad, y utilizando las herramientas más adecuadas de todas las disponibles; asimismo, que reconozca la necesidad del trabajar en equipo con los distintos especialistas implicados en el tratamiento de la obesidad.

Con esta obra -didáctica y práctica- los autores pretenden contribuir a satisfacer la necesidad urgente de que todo individuo que padezca obesidad y que se acerque a los sistemas de salud públicos y privados sea atendido de la mejor manera de acuerdo con las mejores estrategias disponibles en la actualidad. 